\title{
Artificial Neural Networks Application to Calculate Parameter Values in the Magnetotelluric Method
}

\author{
Andrzej Bielecki ${ }^{1}$, Tomasz Danek ${ }^{2}$, \\ Janusz Jagodziński ${ }^{1}$, and Marek Wojdyła ${ }^{3}$ \\ ${ }^{1}$ Institute of Computer Science, Jagiellonian University, \\ Nawojki 11, 30-072 Kraków, Poland \\ 2 Department of Geoinformatics and Applied Computer Science, \\ Faculty of Geology, Geophysics and Environmental Protection \\ AGH University of Science and Technology, \\ Al. Mickiewicza 30, 30-059 Kraków, Poland \\ ${ }^{3}$ Geophysical Exploration Company \\ Jagiellonska 76, 03-301 Warszawa, Poland \\ bielecki@softlab.ii.uj.edu.pl
}

\begin{abstract}
In this paper a possibility of neural network application to data processing in magnetotelluric method is studied. The modular neural system, consisting of three multi-layer neural networks, is used for obtaining geoelectric model of lithosphere basing on amplitude and phase MT curves. Cases of two and three flat lithosphere layers are considered.
\end{abstract}

Keywords: magnetotelluric method, multi-layer neural networks.

\section{Introduction}

Magnetotelluric (MT) is one of the most commonly used passive geophysical method. That method apply measuring of fluctuations in natural electric (E) and magnetic $(\mathrm{H})$ fields in orthogonal directions at the surface of the Earth to determining the conductivity structure of the Earth. Apart from its usefulness in deep lithosphere research and hydrocarbon prospecting, the precise knowledge of the conductivity structure is of major importance in several respects. For example, it has been shown 4 that sites of the Earth's surface lying close to conductive edges are the most sensitive sites for the recording of low frequency signals that have been found to precede earthquakes [7].

The fundamental theory of magnetotelluric was first propounded by Tikhonov [6, and Cagniard 2]. Magnetotelluric data, called amplitude and phase sounding curves, are usually obtained by processing of measured time series, which describe electromagnetic variations in particular point on Earth surface. Sounding curves are next interpreted with various inversion method. The final results are geoelectrical cross-sections (or maps) which are usually correlated with geology. 


\section{Data Processing}

As a results of MT measurements time series of electric (Ex, Ey) and magnetic (Hx, Hy) components are calculated to spectral domain using Fast Fourier Transformation. Such components are connected by formula which includes impedance tensor $[\mathrm{Z}]$ :

$$
E=Z H
$$

where

$$
Z=\left[\begin{array}{ll}
Z_{x x} & Z_{x y} \\
Z_{y x} & Z_{y y}
\end{array}\right]
$$

For horizontally layered Earth $Z_{x x}=Z_{y y}=0$ and $Z_{x y}=-Z_{y x}$ (Cagniard model), so we can obtain magnetotelluric amplitude and phase curves by:

$$
\begin{aligned}
& \rho(f)=\frac{0.2}{f}|Z|^{2} \\
& \phi(f)=\operatorname{Arg}(Z)
\end{aligned}
$$

\section{Neural Approach}

The applied modular neural system consists of three multi-layer artificial neural networks (ANNs). The first one - ANN1 - recognizes how many layers constitute the studied fragment of lithosphere - only two-layer and three-layer cases are considered. If the number of lithosphere layers is equal to two then input vector is put into the second neural network ANN2. Otherwise it is put into the third neural net ANN3. Networks ANN2 and ANN3 calculate parameters of one-dimensional model.

Two various form of the input vector components $\left[x_{1}, \ldots, x_{n}\right]$ for ANNs were tested. In the first case vector components were scaled using the following forms

$$
\begin{gathered}
x_{2 i-1}=\frac{\ln \varrho\left(\omega_{i}\right)}{k_{1}} \\
x_{2 i}=\frac{\phi\left(\omega_{i}\right)}{k_{2}},
\end{gathered}
$$

where $\omega$ was frequency in logarithmic scale, $\varrho$ was resistance, $\phi$ was phase, $k_{1}$ and $k_{2}$ were constant values, calculated during data preprocessing in such a way that components of input vectors $x_{i}$ belonged to interval $[0,1], i=1, \ldots, 55$. In the second case integrated form of input data were used

$$
\begin{gathered}
x_{2 i-1}=\frac{\int_{\omega_{i}}^{\omega_{i+1}} \varrho\left(\omega_{i}\right) d \omega}{k_{3} \int_{\omega_{1}}^{\omega_{5}} \varrho\left(\omega_{i}\right) d \omega} \\
x_{2 i}=\frac{\int_{\omega_{i}}^{\omega_{i+1}} \phi\left(\omega_{i}\right) d \omega}{k_{4} \int_{\omega_{1}}^{\omega_{55}} \phi\left(\omega_{i}\right) d \omega},
\end{gathered}
$$

where $i=1, \ldots, 54$ and constant values $k_{3}$ and $k_{4}$ were used for component normalization as in the case of input data representation given by formula (5). 


\section{Results}

Mean absolute percentage error (MAPE) was used as a measure of accuracy both in the net ANN2 calculating parameters for two-layer model and in the net ANN3 used for three-layer model. The error is given by the formula

$$
\text { MAPE }=\frac{100}{n} \sum_{i=1}^{n} \sum_{j=1}^{m}\left|\frac{y_{i j}-d_{i j}}{d_{i j}}\right|,
$$

where $y_{i j}$ is the $j$ th component of output vector of an ANN if the $i$ th input vector is presented, $d_{i j}$ is the $j$ th component of correct output vector if the $i$ th input vector is presented, $m$ is the number of output vector components and $n$ is the number of presented examples.

Both ANN2 and ANN3 were perceptrons having one hidden layer. In both cases it turned out that twenty hidden neurons was an optimal number. Sigmoidal mapping was used as an activation function of hidden neurons and both sigmoidal and linear neurons were tested in the output layer - see Tables 1 and 2. Components of output signals were scaled so they belonged to interval $[0,1]$. Back-propagation and momentum were used as training algorithms. Both scaled representation of input data (see formula (5) ) and integrated representation (formula (6) ) were tested. Various learning constatnts were tested as well. For both networks (ANN2 and ANN3) training process consisted of 8000 epochs.

In two-layer case the training set consisted of 1072 elements and testing set had 358 elements. Results are presented in Table 1.

Table 1. Results for two-dimensional model

\begin{tabular}{||c|c|c|c|c|c|c||}
\hline No & Data & $\begin{array}{c}\text { Activation } \\
\text { function } \\
\text { for output } \\
\text { layer }\end{array}$ & $\begin{array}{c}\text { Training } \\
\text { parameter }\end{array}$ & $\begin{array}{c}\text { Training } \\
\text { method }\end{array}$ & $\begin{array}{c}\text { MAPE for } \\
\text { testing } \\
\text { set }\end{array}$ & $\begin{array}{c}\text { MAPE for } \\
\text { the whole } \\
\text { set }\end{array}$ \\
\hline 1 & scaled & sigmoidal & 0.2 & backprop. & 3.30 & 3.06 \\
\hline 2 & scaled & linear & 0.2 & backprop. & 2.32 & 1.98 \\
\hline 3 & scaled & sigmoidal & 0.4 & backprop. & 2.53 & 2.48 \\
\hline 4 & integ. & sigmoidal & 0.2 & backprop. & 7.32 & 7.34 \\
\hline 5 & integ. & linear & 0.2 & backprop. & 5.68 & 6.16 \\
\hline 6 & scaled & sigmoidal & 0.8 & backprop. & 3.23 & 3.18 \\
\hline 7 & scaled & linear & 0.2 & momentum & 1.73 & 1.65 \\
\hline 8 & scaled & sigmoidal & 0.4 & momentum & 2.41 & 2.42 \\
\hline
\end{tabular}

In three-layer case the training set consisted of 1147 elements and testing set had 383 elements. Results are presented in Table 2.

The net ANN1, recognizing to which model (two- or three-layer) belonged given curves, consisted of five neurons in the hidden layer and two output neurons. If the first component of output signal was greater then the two-layer model was chosen. The network achieved $100 \%$ of correctness after 700 training epochs. 
Table 2. Results for three-dimensional model

\begin{tabular}{||c|c|c|c|c|c|c||}
\hline No & Data & $\begin{array}{c}\text { Activation } \\
\text { function } \\
\text { for output } \\
\text { layer }\end{array}$ & $\begin{array}{c}\text { Training } \\
\text { parameter }\end{array}$ & $\begin{array}{c}\text { Training } \\
\text { method }\end{array}$ & $\begin{array}{c}\text { MAPE for } \\
\text { testing } \\
\text { set }\end{array}$ & $\begin{array}{c}\text { MAPE for } \\
\text { the whole } \\
\text { set }\end{array}$ \\
\hline 1 & scaled & linear & 0.2 & backprop. & 4.18 & 3.57 \\
\hline 2 & scaled & sigmoidal & 0.2 & backprop. & 4.02 & 3.40 \\
\hline 3 & scaled & linear & 0.2 & momentum & 3.89 & 3.77 \\
\hline 4 & integ. & linear & 0.2 & backprop. & 4.79 & 4.46 \\
\hline 5 & scaled & sigmoidal & 0.4 & backprop. & 4.50 & 4.10 \\
\hline 6 & integ. & sigmoidal & 0.4 & backprop. & 4.40 & 4.13 \\
\hline 7 & integ. & linear & 0.2 & momentum & 4.72 & 4.53 \\
\hline
\end{tabular}

\section{Concluding Remarks}

The presented investigations concerns possibility of artificial neural systems applications in MT method. It should be stressed that presented tests are preliminary and 2D and 3D cases must be studied. Anyway the obtained results are promising and can be a contribution to studies concerning efficiency of modular neural systems ([1], 3], [5]).

\section{References}

1. Auda G., Kamel M.: Cooperative modular neural networks for pattern recognition. Pattern Recognition Let., Vol. 18 (1997) 1391-1398

2. Cagniard L.: Basic theory of the magnetotelluric method of geophysical prospecting. Geophysics, Vol. 18 (1953) 605-637

3. Marciniak A., Korbicz J.: 2000, Modular neural networks. In: Duch W., Korbicz J., Rutkowski L., Tadeusiewicz R. (eds.): Biocybernetics and Biomedical Engineering. Warszawa, EXIT (2000) 135-178

4. Sarlis, N., Lazaridou, M., Kapiris, P., Varotsos, P.: Numerical model of the selectivity effect and the DV/L criterion. Geophysics Research Letters, Vol. 26 (1999) 3245-3248

5. Sharkey A. (ed.): Combining Artificial Neural Nets: Ensemble and Modular MultiNet Systems. Springer, Berlin (1999)

6. Tikhonov, A. N., 1950, The determination of the electrical properities of deep layers of the Earth's crust. Dokl. Acad. Nauk. SSR, Vol. 73 (1950) 295-297

7. Varotsos, P., Alexopoulos, K., Nomicos, K., Lazaridou, M., 1988, Official earthquake prediction in Greece. Tectonophysics, Vol. 152 (1988) 193-196 